\title{
Automated in-core image generation from video to aid visual inspection of nuclear power plant cores
}

\author{
Paul Murray*, Graeme West, Stephen Marshall, Stephen McArthur \\ Dept. Electronic and Electrical Engineering, University of Strathclyde, 204 George Street, Glasgow, \\ G1 1XW corresponding author* email: paul.murray@ strath.ac.uk phone: +44 (0) 1415482502
}

\begin{abstract}
-
Inspection and monitoring of key components of nuclear power plant reactors is an essential activity for understanding the current health of the power plant and ensuring that they continue to remain safe to operate. As the power plants age, and the components degrade from their initial start-of-life conditions, the requirement for more and more detailed inspection and monitoring information increases. Deployment of new monitoring and inspection equipment on existing operational plant is complex and expensive, as the effect of introducing new sensing and imaging equipment to the existing operational functions needs to be fully understood. Where existing sources of data can be leveraged, the need for new equipment development and installation can be offset by the development of advanced data processing techniques.
\end{abstract}

This paper introduces a novel technique for creating full $360^{\circ}$ panorama images of the inside surface of fuel channels from in-core inspection footage. Through the development of this technique, a number of technical challenges associated with the constraints of using existing equipment have been addressed. These include: the inability to calibrate the camera specifically for image stitching; dealing with additional data not relevant to the panorama construction; dealing with noisy images; and generalising the approach to work with two different capture devices deployed at seven different Advanced Gas Cooled Reactor nuclear power plants. The resulting data processing system is currently under formal assessment with a view to replacing the existing manual assembly of in-core defect montages. Deployment of the system will result in significant time savings on the critical outage path for the plant operator and will result in improved visualisation of the surface of the inside of fuel channels, far beyond that which can be gained from manually analysing the raw video footage as is done at present.

Keywords- Visual inspection, image stitching, nuclear power generation, nuclear safety.

ABBREVIATIONS

AGR Advanced Gas-cooled Reactor

SIFT Scale-Invariant Feature Transform

RANSAC RANdom SAmple Consensus 


\section{INTRODUCTION}

Many of the world's nuclear power plants are approaching, or have exceeded their initial design lifetimes. These initial lifetimes were based on conservative estimates of degradation, and there is the opportunity to extend the operational lifetimes of these plants. In the US for example, there is the Light Water Reactor Sustainability (LWRS) program which is looking to support the operation of the fleet of light water reactors to 60 years and beyond. In the UK, several of the $2^{\text {nd }}$ generation Advanced Gas-Cooled Reactor (AGR) power plants have been granted lifetime extensions, with a target of an average of 7 years across the fleet [1]. As the plant components age, there is an increasing need to understand their health and to predict how they will look in the future to ensure continued safe operation.

Two main methods of providing information to support the case for lifetime extension are 1) through condition monitoring, where data relating to the health of the plant is gathered during routine operation, and 2) through inspection, where the plant is shut down and a portion of it is inspected. As well as the safety requirements, there are financial implications associated with outages, as each day a UK AGR is operational, equates to approximately $£ 0.5 \mathrm{M}$ in income.

In the case of the AGR plants, the graphite core is a life-limiting component (along with the boiler systems), and inspection of fuel channels provides insight into the condition of the core. Each reactor consists of around 300 fuel channels and, at the start of life, only a few fuel channels (typically four) were inspected. In 2015 this number has risen to 31 for the oldest plants and the channels to be inspected are selected based on various competing criteria [2]. This results in an increase in the volume of data that needs to be assessed before the plant is returned to service, and this assessment lies on the critical outage path. As a result, the application of automated or semi-automated techniques to aid the processing and analysis of this data in a robust, repeatable, and auditable manner has significant value. In 
future, this will also facilitate an increased number of channel inspections which could positively impact any decisions based on economic factors associated with plant inspection.

One approach for improved data handling would be to design and build new inspection tools which optimize data capture and automatically process the data acquired to provide sophisticated decision support. However, the development and deployment of new inspection equipment, particularly that which is to be inserted into the harsh environment of a reactor core, is very expensive. Leveraging data captured using existing equipment is therefore desirable as it minimizes additional financial outlay. However, the feasibility and complexity of this approach is determined by the constraints of the existing data capture process which may not align directly with the proposed use of the data.

This paper describes the application of advanced signal and image processing techniques to existing in-core inspection TV footage, with the aim of producing chanoramas - full $360^{\circ}$ panoramas of the inside of the fuel channels. The contributions made in this paper include:

1. integrating the use of a-priori knowledge about the camera pose and position to overcome issues of the largely featureless surface areas of the graphite core which restricts the application of more traditional image stitching techniques [3-8]

2. generating these novel chanoramas using data from across the nuclear fleet in the UK in a reliable, repeatable and timely manner, with minimal human intervention

3. dealing with variation in: camera orientation, illumination, noise and other distortions which arise from a data capture system with the absence of the ability to calibrate the camera

4. deployment of software which takes a channel inspection video as an input and automatically generates a chanorama of the inspected channel as an output, without the need for manual intervention. 
More generally, a key contribution of this paper is that we present a technique which makes significantly better use of an existing data set. The proposed approach allows existing inspection videos to be rendered and viewed as a single image where the entire fuel channel inspection video can be seen in the context of the channel itself. The techniques proposed herein can be applied to any video which is captured in a similar format to that used to capture the videos used in this study.

The remainder of this paper is laid out as follows: Section 2 describes: the formation of the AGR cores; the existing inspection techniques; and manual methods for data analysis as well as highlighting the challenges for automating the image processing. Section 3 provides a brief summary of related work and, in Section 4, our new system for converting inspection videos into chanoramas is described in detail. In Section 5 we discuss the results of applying our techniques to process a number of videos and we compare our images to those produced for the same channel using the existing manual techniques before concluding in Section 6.

\section{INSPECTION OF ADVANCED GAS-COOLED REACTOR CORES}

Seven of the nine nuclear power plants in operation in the UK are of the AGR design. The AGR graphite core is constructed from layers of cylindrical graphite bricks, and each layer consists of around 300 fuel bricks plus interstitials which are physically keyed together. Cores generally consist of 11 brick layers, although this varies from plant to plant, and each of the bricks stands around $800 \mathrm{~mm}$ tall. The graphite core is configured in this way to form various channels to accommodate fuel, control rods and control the passage of the $\mathrm{CO}_{2}$ gas coolant. As well as providing the physical structure for housing the fuel, the core also acts as a neutron moderator. Under fast neutron irradiation and through the process of radiolytic oxidation, the properties of the graphite bricks, such as their dimensions, strength and Young's modulus all change, and under certain conditions the bricks can crack. Tracking these changes and their effects through regular monitoring and inspection of the core [9-11] 
can provide supporting evidence that indicates the cores are in good condition and remain fit for purpose.

\subsection{Fuel channel inspection and video capture}

During an outage, part of the inspection process uses tools named CBIU (Channel Bore Inspection Unit) and NICIE (New In-Core Inspection Equipment) which are lowered into each of the AGR fuel channels selected for inspection [12]. These tools are equipped with various sensors including, a video camera, which are used to acquire data from inside the channel. Using the tool to record videos of successive vertical scans of the channel wall at different camera facings with sufficient overlap between the scans ensures that full coverage

of the internal channel surface is obtained. During these scans, detailed measurements of the channel bore are also recorded. If anything unusual is observed during these scans, such as a defect in the channel wall, the region of the channel that contains the defect is revisited, and additional video data known as "crack following footage" is recorded. This additional footage is gathered solely for the purpose of manually generating montages which are used to assess and quantify the defect, and no further bore measurements are taken.

\subsection{Existing Data Analysis Techniques}

At present, all data analysis is performed manually by an expert operator. As a result, the existing manual montage generation process is time-consuming and labor-intensive, requiring suitable frames to be identified and extracted from the footage and manually assembled into the montage using third party image manipulation software. The resulting montages are assessed along with other measurements taken during the inspection to make a qualified statement to support return to service of the plant. The method proposed in this paper for automatically generating chanoramas from just the vertical scans: speeds up the generation of crack montages; provides additional images of the surrounding area; and removes the need to re-visit and record the additional crack following footage. This means better crack montages are generated automatically in a shorter time than is required to produce them manually by 
the existing approach. Furthermore, the automatic approach requires less data to be captured thus reducing the channel inspection time. This in turn shortens the plant outage duration.

\subsection{Video format and challenges for automatic processing}

The inspection video consists of a series of vertical scans of the channel at (generally 6) different orientations. Since the camera has a $70^{\circ}$ field of view, these orientations provide a small amount of horizontal overlap of the scans in the spatial domain and allow the entire channel to be visually inspected using the camera. At first, automatically processing the video to produce a stitched image may seem like a trivial task. However, there can be many variations in the way in which the data is recorded including: changes in illumination and in camera pose; differences in the order in which the camera is rotated; changes in the scan direction and in optical configuration; variation in the number of scans recorded; as well as changes in the physical location of the tool at the beginning and end of each scan. This is not to mention the presence of image noise and, a lack of distinctive features for use when aligning images before stitching. The variations in the data format as described often occur between plants but, in some cases, variations do occur within a set of videos from a single inspection of one plant. In fact, it is not uncommon to observe illumination changes, and variations in tool position at the beginning and end of each scan within a single fuel channel inspection itself. All of these factors present a significant challenge for implementing a system capable of automatically processing data from all channels at each plant to convert videos into static images. While a protocol could be implemented for all future data acquisition, this clearly cannot be applied to data which has already been recorded and the proposed system must therefore work on historic data in its existing format. In fact, even if a protocol is prescribed for future data capture, there is no guarantee that it will be followed during data acquisition as various factors can affect the process during channel inspection. 
3 RELATED WORK

The creation of large montages from multiple images is a technique which is commonplace in consumer photo editing products [8]. The approach to stitching together a series of images to form a composite image can be described conceptually by:

$$
I_{o}=\lambda D+(1-\lambda) K
$$

Where $I_{\mathrm{o}}$ is the output image, $D$ is the application of domain-independent image stitching techniques, $K$ is the a-priori knowledge which can be factored in and $\lambda$ is a value between 0 and 1 representing the balance of these two factors. A $\lambda$ value of 1 represents no a-priori knowledge is used in the image stitching process. As $\lambda$ decreases, more domain specific knowledge is utilised by adding constraints on how each sub-image can be stitched in the global image (potentially reducing the calculation overhead). Examples of a-priori knowledge would include, knowing that all images are taken at the same scale or that they are all oriented the same way. Or, if some information about the motion path of the acquisition device is available, more advanced relationships between overlapping images can be approximated. At the other end of the spectrum, a $\lambda$ value of 0 means the exact location, rotation and scale of each sub-image is known and no explicit additional image transformations need be calculated.

Central to the generation of panoramic images is the need to correctly align the individual overlapping component images before seamlessly blending them together. In situations where there is no a-priori knowledge $(\lambda=1)$, the alignment between overlapping images can often be estimated from the data itself. There are two main approaches to determine the alignment of images for automated approaches and these are known in the literature as the direct approach and the feature based approach [8]. The direct approach performs a comparison of image pixels in overlapping frames to deduce the geometric relationship, whereas the feature based approach extracts key features from both images and uses matching features to estimate 
alignment prior to stitching. For both methods, it is important that there is sufficient detail in the images to be aligned. If there is a lot of detail in the images, it is likely that there will be some distinct features in both of the overlapping images, which can be used to determine image alignment. Unfortunately this is rarely the case when considering images of the surface of fuel channel bricks, which tend to be large, grey, featureless surfaces. This issue also arises in other industrial environments in which visual inspections are performed, e.g. pipes and storage vessels in the oil and gas sector [13-15]. In such cases, it is often difficult to find enough distinct feature points in overlapping images that can be used to accurately estimate image alignment prior to stitching. In the next section, we assess the applicability of the direct and feature-based approaches and contrast them with a knowledge-based approach, which makes assumptions about the position of the camera $(\lambda=0)$ to generate a montage.

\section{CONVERTING IN-CORE INSPECTION VIDEOS INTO STATIC IMAGES}

In our application, there is some knowledge about the data capture process used when recording each video. For example, the direction of the camera motion is generally known, the tool speed is assumed to be constant, and the camera facing for each vertical scan of the channel (the camera orientation sequence) can be logged during data capture. There is also a small amount of metadata embedded within the video in the form of overlay text which is shown in a frame extracted from one video in Figure 1(a). The video overlay provides information such as, the name of the plant and channel being inspected, the date and time of inspection, the tool height within the channel and the camera facing for each vertical traversal of the channel. Provided the aforementioned information is available, automatically creating a chanorama is a five-stage process: 
1. the raw video footage is converted into frames

2. the frames are analysed to find the beginning and end of each scan - all frames which do not belong to a vertical scan are then discarded

3. individual montages of each vertical scan are constructed

4. the resulting vertical scan montages are aligned with each other in a global image

5. the vertical scan images (generally 6) are stitched together to create a chanorama of the full fuel channel surface.

The video to frame conversion can be achieved using any open source or commercially available software for this task. As such, the details of this step will not be discussed further in this paper. Instead, we now focus on explaining the sophisticated signal and image processing techniques we have designed to analyse the frames extracted from the video in order to automatically create chanormas.

\subsection{Identifying frames corresponding to the beginning and end of scans}

When the inspection tool is stationary, the section of the image containing information about the tool height in the video overlay does not change since the tool is not moving. However, when the tool is being translated vertically within the channel, the tool height (obviously) changes, and so does the section of the image displaying this information in the overlay. The region of the image which contains the tool height information is highlighted in Figure 1(a). A simple solution would be to analyse this height data to identify the individual channel scans based on rates of change or some other suitable technique. However, the height data is not recorded separately and the only way to obtain it would be to use Optical Character Recognition [16] to extract it from individual frames in the video.

A simpler solution is therefore to extract the region of the image containing the tool height value from each frame in the video and threshold it (to convert it to binary) [17], before computing and storing the sum of absolute differences between each of these regions in 
successive frames. If applied to an entire video sequence, this process produces a video summary profile like the one shown in Figure 1(b).

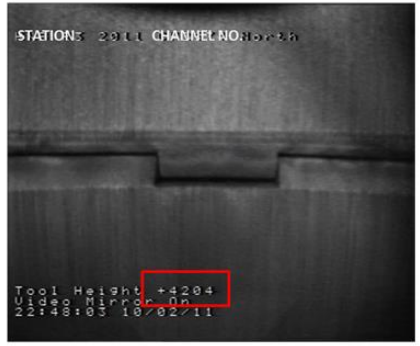

(a)

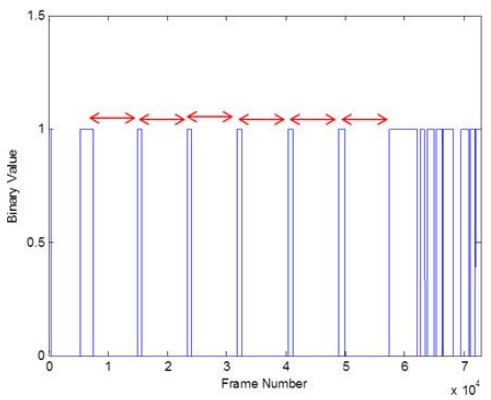

(d)

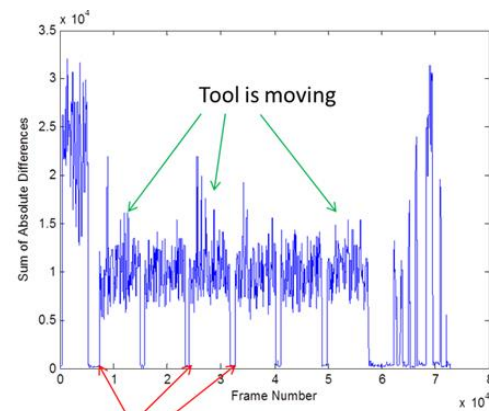

(b)

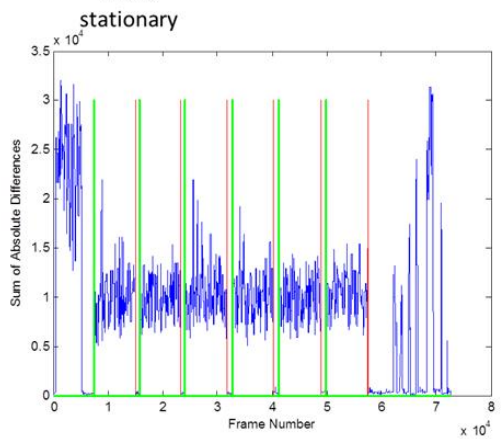

(e)

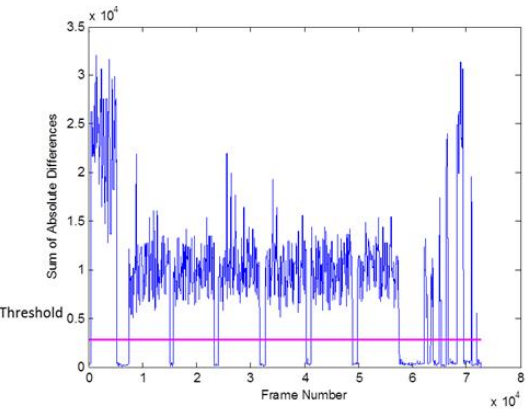

(c)

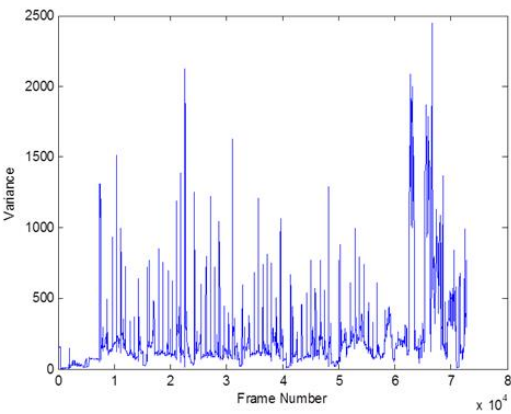

(f)

Figure 1 Detecting the beginning and end of each scan (a) Image with tool height information highlighted (b) Video summary profile (c) Video summary profile and threshold value overlaid (d) Binary signal where "ON" means tool is stationary - red arrows highlight points which are separated by the expected distance of a scan (e) Start points (green impulses) and end points (red impulses) overlaid on video summary profile (f) Variance profile for the same video.

In practice, not all frames need to be used to create a video summary profile. In fact, the one shown in Figure 1(b) was computed using every 20th frame to reduce processing time. In the video summary profile, relatively high values (large differences) indicate that the tool is moving, and, conversely, relatively low values (little differences) indicate that the tool is stationary. By automatically analysing video summary profiles like the one shown in Figure 1(b), it is possible to identify frames in which the tool starts and stops moving and, subsequently, to detect the frames which correspond to the beginning and end of each scan. The first step in the automatic analysis is to smooth the video summary profile before thresholding $[17,18]$ it to extract local minima corresponding to frames in which the inspection tool is stationary. This concept is demonstrated in Figure 1(c) which shows the video summary profile and the computed threshold value. In Figure 1(d), the result of 
applying this threshold is shown where "on" values indicate the tool is stationary. By computing the distance between these points in the resultant binary signal shown in Figure 1(d), and searching for those points that are some fixed distance, equivalent to the length of each scan apart, it is possible to extract the frame numbers which correspond to the beginning and end of each scan. This is demonstrated in Figure 1(d) where there are 7 "on" points (pulses) separated by roughly the same fixed distance in the binary signal. If the distance between these points lies within the range of the expected number of frames between each scan in the video, it is likely that these points denote the beginning and end frame of each scan. These frame numbers can therefore be extracted as candidate start and stop points as shown in Figure 1(e).

One drawback with relying solely on the distance between the detected points is that any two stoppages of the tool which happen to be separated by the same distance as that expected for a scan will be detected as such and corresponding start and stop points will be computed. For example, if the time taken for the tool to enter the channel and reach the bottom (while the forward facing camera is recording) lies within the expected time frame for a scan, this region of the video would be detected using the algorithm described. To improve our confidence that the points detected by the algorithm do in fact correspond to the channel scans, a second video summary profile can be computed and analysed. This second summary profile is computed by finding the variance, $\sigma^{2}=\sum_{i=0}^{N} \frac{\left(x_{i}-\mu\right)^{2}}{N}$, of pixel intensities within a small window (corresponding to the pixels which change between frames) of successive frames, where $x_{i}$ denotes an image pixel, $\mu$ is the mean of the image and $N$ is the number of pixels in the image. The variance profile for the channel used here for demonstration is shown in Figure 1(f).

Spikes in the variance profile correspond to regions of change e.g. brick interfaces (where two bricks stacked on top of each other meet - see Figure 2, Figure 3 and Figure 4) which are 
generally darker than their surroundings. In situations where more than the desired number of scans is detected, the scans with the fewest peaks in the corresponding region of the variance profile are rejected. Rejecting candidate start and stop points based on the number of peaks in the variance profile (assuming that a scan should contain brick interfaces) allows the correct start and stop points to be retained for further processing.

\subsection{Stitching vertical strips for channel scans}

The first step in processing the individual frames of the video is to remove the overlay text since this should not be allowed to affect the results. When the overlay has been cropped from the source image, this leaves us with a frame size of 620 by 410 pixels. The large number of frames generated from the video, with a high degree of similarity between consecutive frames, offers a range of options for constructing a montage depending upon how much knowledge is utilised in the stitching process. If the assumption is made that the camera moves at a fixed rate and at a fixed orientation, which is reasonable given the equipment set up, then the distance moved between successive frames is constant. In our application, the speed of the camera hoist equates to a vertical shift of 5 pixels between successive frames.

To consider the situation where our assumption of fixed rate and orientation of the camera was incorrect, we wished to explore the possibility of using either the direct or feature based approach (see [8] for more details) for the construction of the montage. However, applying these stitching methods to all the frames immediately caused an issue, as each frame contains a slightly different view of the same scene where a) the object is not flat, but the inside of a cylinder and b) is illuminated in a non-uniform way. This is illustrated in Figure 2 which shows a sequence of three frames showing the same feature, a brick interface, taken from the same small piece of footage. In the first image, the brick interface is slightly above the centre of the camera in the field of view and is bowed upwards, in the second image the camera and interface are directly aligned and in the third image the camera is slightly above the interface 
in the field of view causing a downward bow. Attempting to stitch these three frames to form a composite image using the standard direct and feature based approaches fails to produce a satisfactory result, as the curvature of the channel is not taken into consideration. One approach to tackle this would be to perform a calibration operation on the camera [19]. Techniques for performing this task tend to introduce calibration images or known patterns into the scene. These are then imaged and used to learn mathematical transformations which model distortions introduced by the optics of the imaging system and, in this application, the curvature of the channel. However, as no access to the camera was/is available, traditional techniques for this task were not pursued further.
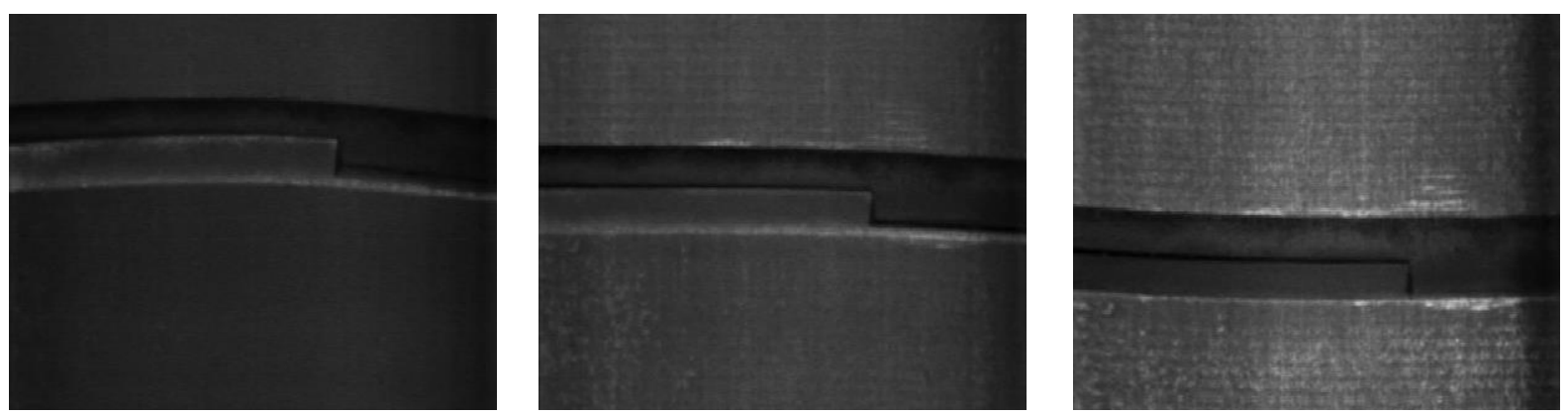

Figure 2 Screenshots of the same brick interface at three different instances, indicating the effect of the curvature of the fuel channel wall

\subsubsection{Full frame abuttal montage generation}

If the assumption of fixed rate and position of the camera was made, and given that there is 5 pixels vertical shift between successive frames then, given a frame height of 410 pixels, every $82^{\text {nd }}$ frame could simply be abutted to form a panorama of the channel. This offers the benefit that the minimum number of frames needs to be extracted from the video and assembled to form the montage. A typical output using this approach is shown in Figure 3(a) where stitched images from each camera orientation have been aligned and placed side by side to show a single brick layer containing a crack. The image quality of the output produced by this approach is poor and it is clear that a series of images have been abutted one on top of the 
other. The undesirable effects of lens distortion and non-uniform illumination are also clearly visible in the final result achieved when using this approach.

\subsubsection{Window extraction and abuttal montage generation}

Since the image data is acquired at a rate that is much higher than the speed at which the camera traverses the channel, there is significant overlap between successive frames. As a result, the effects of non-uniform illumination and lens distortion across the image shown in Figure 3(a) can be suppressed by cropping a much smaller window from each and every frame rather than abutting a large section from every $n$th frame. Additionally, by selecting the position of this window to coincide with the point at which the interface and camera align has the effect of practically eliminating the "bowing" artefacts caused by lens distortion and the curvature of the channel.

This process is illustrated in Figure 3(b) where the height of the window of pixels cropped from each frame has been set by the speed of the camera hoist. Building the output image by stacking small regions cropped from each successive frame generates a high quality image like the one shown in Figure 3(c) and no blending or other correction process is required to produce the final result. Furthermore, the effects of lens distortion are less apparent and the non-uniform illumination is suppressed - particularly in the vertical direction. Alleviating the need for pre/post processing in this way reduces the time taken to produce stitched images which are representative of the channel bore without introducing false pixel values in the blending/correction process. One drawback is that this approach does not account for the possibility of sub-pixel motion between frames. Instead, we assume that the appropriate window height which should be extracted from each frame corresponds to an integer number of pixels. For the application described in this paper, this assumption has been demonstrated to be valid as shown by the image in Figure 3 (c). 


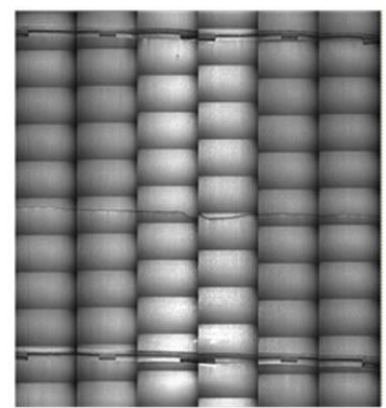

(a)

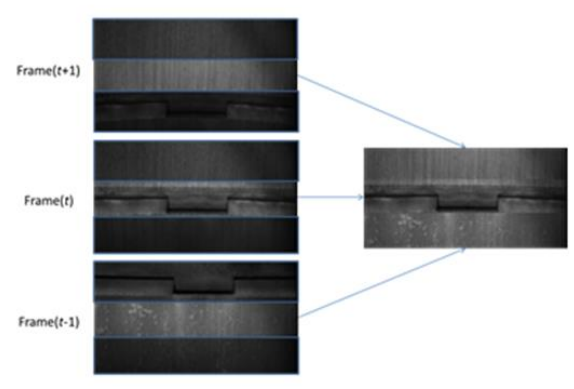

(b)

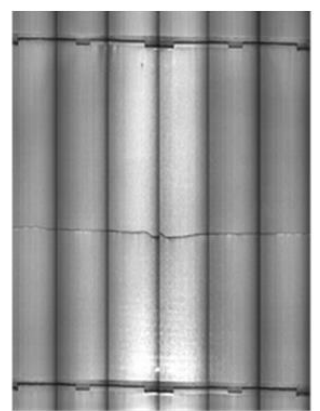

(c)

Figure 3 Example of generating 2D stitched image from sequential frames captured during a bottom to top scan from inside the fuel channel. (a) Image produced by abutting non-overlapping frames in global output image (b) Video sequence ( 3 frames) from which a small region of each frame is extracted and placed in the global output image. (c) Result of building output image of a single brick layer using a 5 pixel high window from the centre of each frame.

\subsubsection{Fixing the window height parameter}

The camera moves in the vertical direction, therefore, only the height of the window needs to be defined so that the region which is cropped from the image corresponds directly to those pixels which have changed between successive frames. In the case of the approach described here in Section 4.2.2, the window height is fixed by a user based on the speed at which the camera moves when acquiring the data. While this method provides a robust and efficient way to create montages of the individual scans of the channel, we also wanted to test how well more traditional image stitching techniques would perform. For this reason, a direct and feature based approach were developed and used to compute a suitable value for the window height based on the estimated image alignment between successive frames. The application of these direct and feature based approaches was undertaken for comparison purposes and to demonstrate robustness against variations in camera hoist speed and camera orientation.

\subsubsection{A Direct Approach}

After cropping out the overlay text in the video sequence, matching regions in successive frames are identified using cross correlation. Since most of the video contains little detail, an edge image is computed for each successive frame using the Sobel operator [17] and the same threshold is applied to each image when any two frames being compared. This 
generates a random binary pattern of edges from neighbouring images such that these random patterns can be matched using cross correlation. By computing the position of the maximum value in the correlation output, for neighbouring binary images, we can estimate the vertical shift between these patterns in successive frames. This shift equates to the number of pixels which have changed between the current frame and the previous frame and can be used as the window height for producing the stitched strips.

\subsubsection{A Feature-based Approach}

After cropping out the text overlay, features are computed for neighboring images using SIFT (Scale Invariant Feature Transform) [20]. SIFT is a robust algorithm for automatically extracting and describing feature points in image data and is widely used in image stitching applications. For our purposes, SIFT is applied to neighboring frames and matching points between these frames are identified. Outliers introduced during the matching process are then rejected using RANSAC (RANdom Sample Consensus) [21] before the average distance between the remaining, correctly matched points is computed. RANSAC is another widely used algorithm in the image stitching community which repeatedly samples the pairs of matched points and rejects outliers by finding the largest consensus set of points which match the underlying model. The average distance between the remaining matched points (after applying RANSAC) is then used as the window height when cropping regions from the current frame and placing them in the global image to produce the stitched output.

\subsubsection{A Knowledge-based Approach}

Since the camera is known to travel at a constant speed as it is raised from the bottom of the channel to the top, the number of pixels which actually change between successive frames as the camera moves should be constant. With this knowledge, we are able to simply estimate the optimal window parameters such that it contains only the pixels that have changed between the current frame and the previous one and fix this height when stitching the entire video sequence. The optimal window height was estimated empirically to be 5 pixels high by 
measuring (manually using image analysis software) the number of pixels which change between successive frames. For the knowledge approach, no further calculation of window height is required. A 5 pixel high window which spans the width of the image is cropped from each frame and placed in the correct location in the global output image. The window height can be increased or decreased if the camera moves faster or slower respectively. In cases where a camera is known to change speed during capture, a model can be developed to adapt the window height for different sections of the video to avoid repeating or missing data.

\subsubsection{Comparison of approaches}

Each of the implementations described in the previous sections (except the full frame abuttal method) were applied to the same data set in order to assess and compare their performance with frames extracted from the original video. Figure 4 shows crops from the full channel images produced using each of the three methods for stitching the data alongside a frame from the original video sequence of the same part of the channel. This section of the channel contains a vertical bore crack intersecting a brick interface (Figure 4(a)).

Clearly, all three methods accurately reproduce the image of the crack and the brick interface as seen in the original video frame where approximately 116 frames have been used to produce the images shown in Figure 4(b), (c) and (d). Furthermore, there is no visible difference in the image quality produced by each of the three methods. On average, the knowledge-based approach processes a single frame in 9ms when executed on a PC with a Core i7-3770 processor and 16GB of RAM. On the same system, the direct approach takes around $135 \mathrm{~ms}$ per frame and the feature based approach can take up to $500 \mathrm{~ms}$ to compute the height of the window which should be cropped from the current frame. Since there is no observable difference in the images produced by each method, and since the knowledge approach is a fast, effective and robust alternative to the more computationally expensive traditional image stitching techniques, this is the preferred solution for our system. 


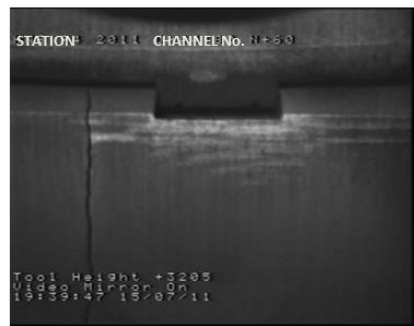

(a)

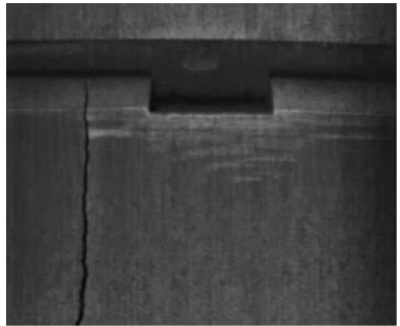

(b)

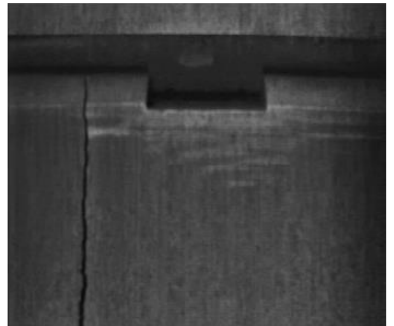

(c)

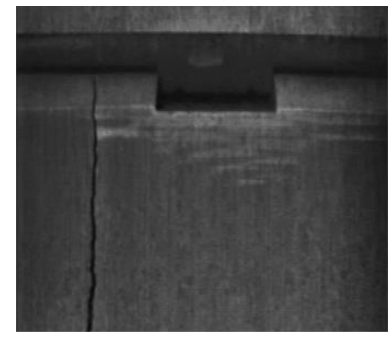

(d)

Figure 4 Comparison of image alignment techniques using 116 frames (a) Original image from video sequence containing a brick interface and a crack. (b) Knowledge approach (c) Direct approach (d) Feature approach

\subsection{Aligning the Strips in a Global Output Image}

When all six vertical strips have been stitched, they are placed side-by-side and aligned vertically in a global image before the horizontal alignment and stitching is performed. Since the vertical strips generally differ in height, it is not sufficient to place them side-by-side without first computing the optimal vertical alignment between them. An example of three bricks cropped from the bottom of a pair of overlapping strips (rotated through $90^{\circ}$ ) is shown in Figure 5, where the misalignment between brick interfaces is clear.

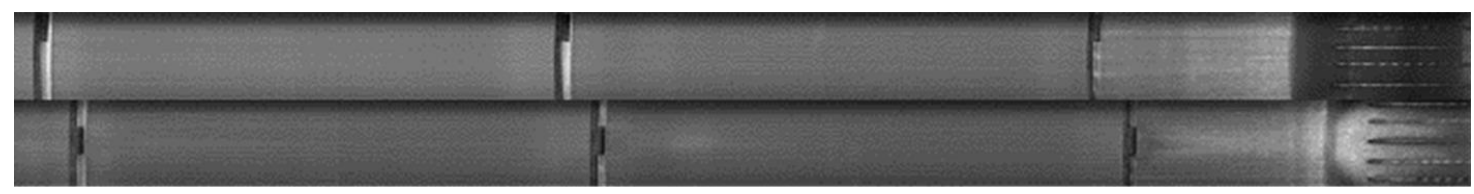

Figure 5 Two regions of overlapping $N$ and $N+60$ scans highlighting the misalignment that exists in the brick interfaces when the images are simply placed side by side.

In order to estimate and compensate for the offset between strips, we firstly apply a Sobel mask, [17], to locate horizontal edges in the overlapping images. This produces a binary edge image for each strip where, in general, the "on" pixels correspond to the brick interfaces. By summing each row, these edge images may each be reduced to a $1 \mathrm{D}$ profile before computing the $1 \mathrm{D}$ cross correlation of these signals. The best alignment between two overlapping images is then easily computed by finding the position of the maximum value in the cross correlation result. The computed offset can then be used to align each overlapping stitched image 
vertically in the global output image. Figure 6 shows the results of applying this method to the two overlapping stitched images shown in Figure 5.

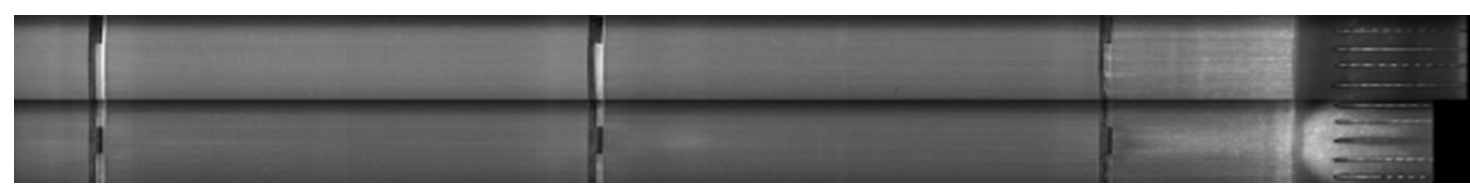

Figure 6 Result of aligning two overlapping images in a global image using the method described in this section.

4.4 Estimating the Horizontal Overlap between Scans

The final stage in the image stitching process is to estimate the extent of the horizontal overlap between neighboring scans before shifting the images left/right accordingly to produce the final chanorama. Since the overlap is not constant between all six scans, and since it depends largely on the engineer who is manually positioning the camera, the knowledge approach cannot reliably be used for this task. Instead, we have used SIFT [20] to extract and match feature points which can be used to estimate the horizontal overlap between overlapping images. SIFT which was explained briefly in Section 4.2.5 was chosen due to its accuracy when applied to the problem.

The algorithm for horizontal stitching when applied to a pair of overlapping images can be summarized as:

- Compute SIFT points from overlapping images of vertical scans

- Perform matching of SIFT points

- Reject outliers using RANSAC

- Estimate the extent of the horizontal overlap

- Crop the right side of the leftmost image by the horizontal overlap

- Shift the rightmost image to the left by the value of the horizontal overlap

As we have explained, a major difficulty for stitching the images captured from inside the channels of the reactor core is that there is a lack of distinctive features in the data. As a result, when performing horizontal image alignment of the vertical strips, very few features 
are extracted, and hence the matches that do occur are often erroneous when two entire strips are being aligned. Since the strips have already been aligned vertically using the cross correlation approach described in Section 4.3, it is possible to extend the application of SIFT by constraining the matching algorithm in order to reduce the likelihood of false matches. For example, we know that points at the top right of one image cannot possibly match points in the bottom left of its aligned neighboring image. To prevent such matches from occurring, the algorithm can be applied to small blocks of each image in turn starting at the top and working through to the bottom. In this heuristic way of constraining SIFT, we are applying the algorithm to small overlapping windows where matches are likely to occur and thus forcing the algorithm to find matches in those regions. This approach is effectively reducing the value of $\lambda$ introduced earlier in Equation (1) of Section 3 in order to make more use of the pre-existing knowledge available about the relationship that exists between overlapping images of the scene.

Each time the algorithm is applied to extract features and find matches, a histogram of the horizontal distance between matched points for that image block is computed after RANSAC has been applied. The result is a number of histograms containing estimates of the horizontal overlap between the subsections of the images. From each histogram, the most frequent estimate is selected as the horizontal overlap. A confidence score for this estimate is computed using:

$$
C S=1-\frac{E[h]}{\max [h]}
$$

where $C S$ denotes the confidence score computed from histogram $h$. The estimate with the highest confidence is then used as the horizontal overlap for the pair of images in question.

In Figure 7 we have shown an example of a single brick layer cropped from a full channel image before and after horizontal stitching. Both images have been processed using the 
stitching technique described in the previous sections and have been aligned using the cross correlation approach described in Section 4.3. The regions circled in Figure 7 illustrate the capability of the horizontal stitching routine.

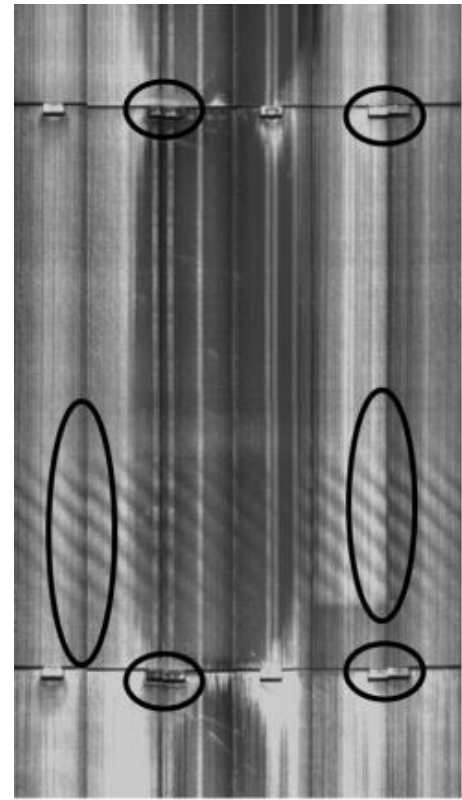

(a)

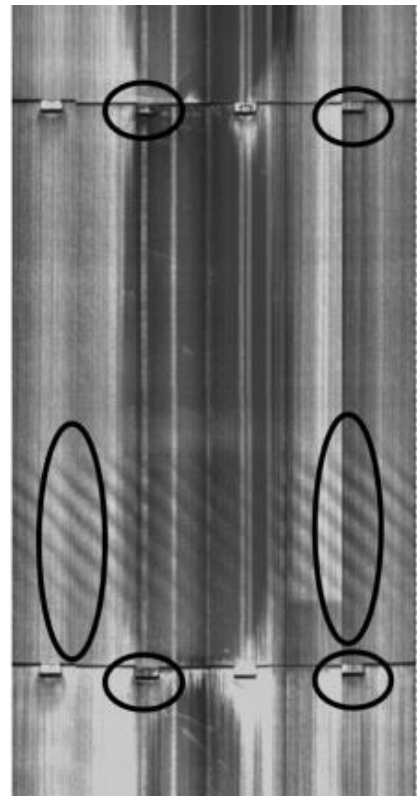

(b)

Figure 7 Example of horizontal stitching (a) Image before horizontal stitching (b) Image after horizontal stitching

\section{RESULTS AND DISCUSSION}

The system described in this paper has been applied to data from all 7 AGR plants in the UK. To date, over 75 full channel images have been generated and the initial results are extremely promising. One of these full channel images, as well as a zoomed region of this montage, and three zoomed regions from different full channel images, are shown in Figure 8 . The image shown in Figure 8(a) represents almost one hour of video footage. Shown here as a snapshot, the inspection footage can be interpreted in a glance, and features like the crack in brick layer 7 (highlighted in the black box in Figure 8(a)), can be identified, located and quantified in the context of the brick, and with respect to the full channel. A zoomed version of this feature is shown in Figure 8(b). It should be noted that the images shown here from inside the reactor 
cores of the AGRs are some of the first of their kind ever to be seen (some of which were first presented in [9]).

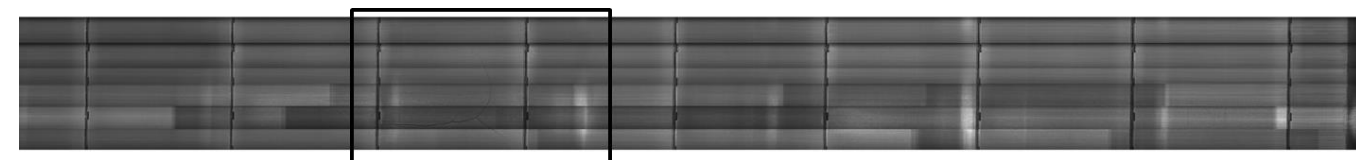

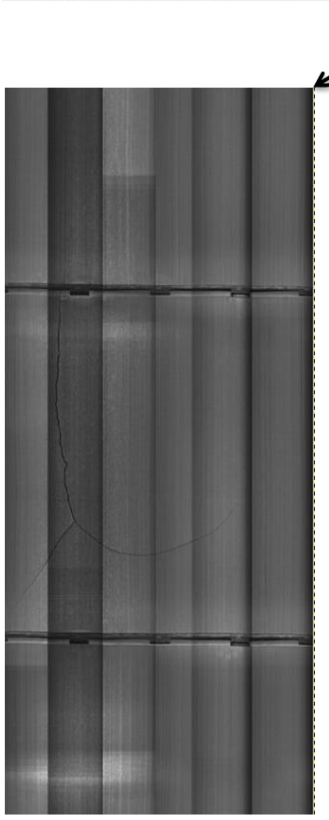

(b)

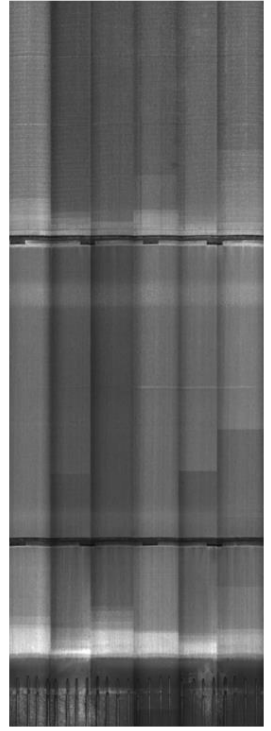

(c)

(a)

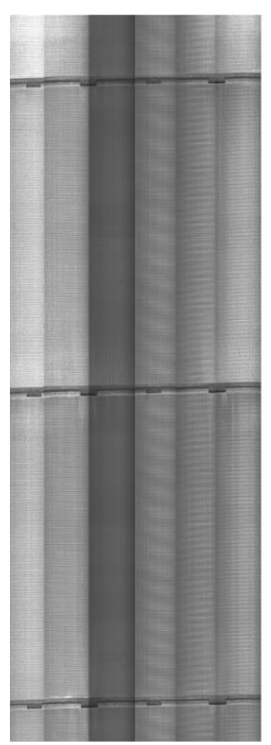

(d)

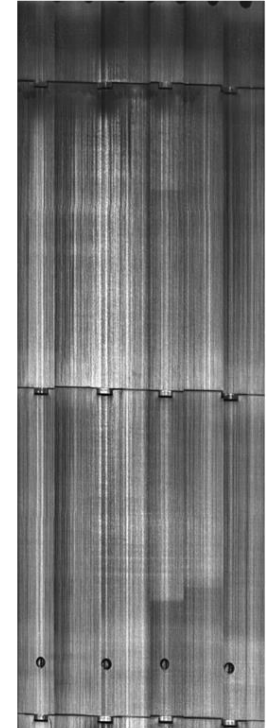

(e)

Figure 8 Some results produced using the method described in Section 4 to produce chanoramas from inside AGRs. (a) Chanorama rotated through $90^{\circ}$ for visualisation (left of page: top of channel and right of page: bottom of channel). (b) Zoomed region of (a) highlighted to show crack. (c) Bottom region of a channel showing 2.5 brick layers and methane holes. (d) Two brick layers from a different channel. (d) Brick layer with trepanned holes.

We have compared the output of the approach described in this paper with an example of a stitched image generated using the existing manual process. The first comparison, shown in Figure 9, is concerned with the level of core coverage offered by the automatic method when compared with the manual approach. While the automatic approach generates a chanorama, the manual approach offers coverage in the region of just $3 \%-4 \%$ of the entire channel. The comparison shown in Figure 10 is concerned with the resultant image quality when using the manual approach, shown in Figure 10(a), and the automatic one, shown in Figure 10(b). 


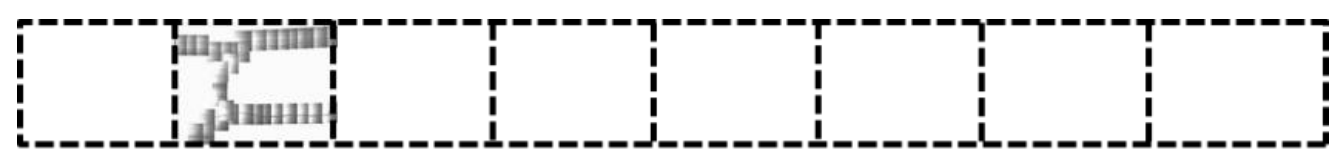

(a)

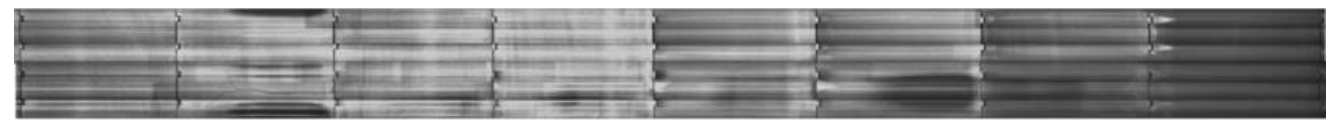

(b)

Figure 9 Comparison of the core coverage offered by the manual and automatic method for image stitching. (a) Core coverage offered by manual approach equates to around $25 \%$ of a single brick layer which is between $3 \%-4 \%$ coverage of the full channel. (b) Chanorama - a full channel image offering $100 \%$ channel coverage.

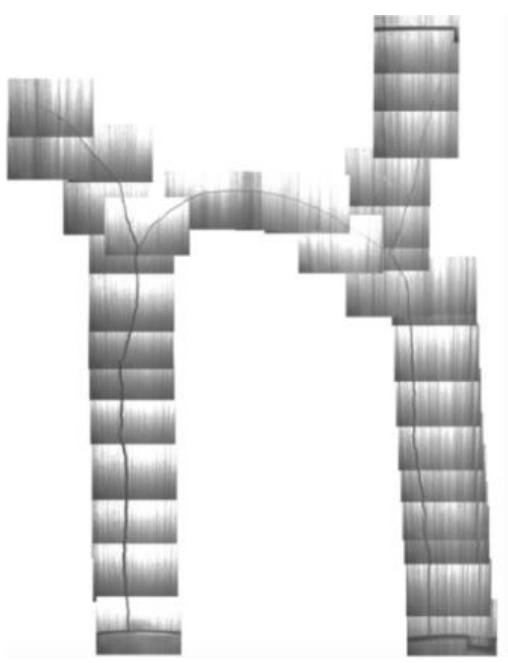

(a)

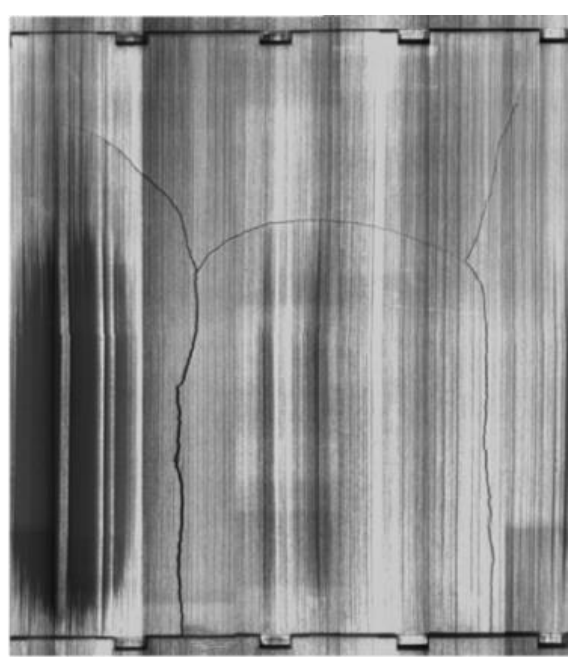

(b)

Figure 10 Comparison of existing approach with proposed approach for image stitching. (a) Stitched image of defect region (produced manually from the crack following footage). (b) Stitched image of defect region cropped from the chanorama (generated automatically from vertical scan footage using proposed approach).

By visual inspection, it is clear that the quality of the image produced by the automatic techniques described in this paper is significantly higher than the quality of the image produced by manual assembly of individual full frames. While both images clearly show the crack, the result produced by the automatic approach offers a higher quality image which contains much more information than the one produced with the existing method.

Figure 9 and Figure 10 demonstrate exactly how better use of all data available has the potential to make inspections more reliable in future. The main reason for improved image 
quality when using the automatic approach, is that by taking small strips from each consecutive frame and stacking these on top of each other, we actually remove the majority of the non-uniform illumination and saturation that is present in each frame of the video. We also reduce the effects of lens distortion retrospectively and without camera calibration data (as this is not available). However, this is not the case when the montages of defect regions are manually assembled using entire frames from the video. In this situation, the non-uniform illumination and saturated pixels are widespread throughout the stitched image.

While it is theoretically possible to manually stitch a full channel image from any video sequence, it is simply not practical to do this. The time required to manually produce images of cracks and defect regions alone, like the one in Figure 10(a), cannot be quantified in advance and can take more than a full working day to complete. This time increases significantly if multiple cracks appear in a single channel since each crack requires a different stitched image to be produced. On the other hand, the automatic approach can be completed within 15 - 30 minutes using state-of-the-art PCs to produce images like the ones shown in Figure 8(a) and Figure 9(b) which offer 100\% channel coverage. This time depends only on the length of the video sequence and hence the number of frames which must be processed. The number of anomalies in the channel does not affect the time taken to automatically produce a full channel image. Furthermore, using this approach will alleviate the requirement to capture crack following footage which would directly reduce the time taken to inspect channels. Longer term, this should allow more channels to be inspected more frequently and could lead to the existing nuclear plants operating for longer to provide a reliable source of energy for the UK.

Due to the minimal time overhead associated with the automatic stitching method, even in situations where no cracks are found in a channel during inspection, it is useful to generate full channel images of fuel channels. These images, like the one in Figure 9(b), would allow 
experts to study images of fuel channels in more detail and to observe various features of the channel like never before. For example, expert operators could view and study images of fuel channels from which samples have been trepanned for testing in the lab to gather data about the state of the graphite in the core. Furthermore, the effects of dose-end-depression caused when the fuel is in-situ and the plant is at power, as well as fundamental features of the graphite itself can also be seen from such images. In addition to this, the images produced following any inspection can be archived and used for reference in future if any cracks are found during a repeat channel inspection. Finally, in situations where other measurements and inspection techniques provide conflicting results about surface defects in a channel, images like the ones presented here can be readily consulted to confirm or deny the existence of any anomaly.

In spite of all the benefits we have discussed, it should be noted that there is still a small amount of non-uniform illumination across each vertical strip image which is generated using the automatic method. This is observable in all of the stitched images shown in this paper which have been produced using the techniques described herein. It is this non-uniform illumination that causes the individual strips to exhibit a "tubing" effect in the final stitched montage. Techniques for removing this non-uniform illumination by using surface fitting are currently under investigation.

\section{CONCLUSIONS}

In this paper, we have presented a novel approach and system for automatically generating stitched images from videos recorded during in-core inspections of fuel channels in the UK's AGR nuclear fleet. We have explained the existing visual inspection and data capture process and we have highlighted the variations in this which pose challenges for designing a system to automatically convert the inspection videos into high quality full channel images of fuel channels within the core. We have provided a summary of existing image stitching 
techniques and explained that on obtaining unsatisfactory results when applying existing state-of-the-art techniques [15] and [13] to our data, we chose to design our own automated image stitching system for processing AGR inspection videos. We have described our method in full and demonstrated the high quality full channel images it is able to produce.

The novel approach for image stitching, which uses knowledge relating to the position and facing of the camera during footage capture has been compared to two other stitching techniques based on a direct approach and a feature based approach for computing alignment. Our knowledge based approach was shown to perform as well as the other approaches in terms of accuracy and image quality, but with the benefits of reduced complexity and computational running time. In section 5, several full channel images from inside the reactor core of the AGR were shown and compared with results produced using the existing manual approach. The full channel images (known as chanoramas) shown here could not practically have been produced without the method described herein. We have explained the existing manual approach that is currently used to produce stitched images of defect regions, and we have demonstrated that in comparison the techniques proposed in this paper offer a significant improvement in terms of efficiency, image quality and channel coverage.

As the AGR plants grow older, there will be increasing pressure to inspect more channels more often to provide supporting evidence that plants are safe and reliable for continued operation. Reducing the time taken to produce stitched montages of defect layers (and the rest of the channel) using the automatic approach described in this paper is of benefit as this activity often lies on the critical path during an outage. Also, if over time channels exhibit multiple defects, the automatic approach (unlike the manual one) will not take any longer to generate all crack montage images required to return plants to power. In fact, time savings like this which lie on the critical path could prevent premature closure of healthy plants by reducing to the operational costs of statutory outages. 
It is also important that the size and condition of any defects in the channel are measured over time and that any changes can be readily quantified. By using the automated image stitching techniques described in this paper to produce full channel images, the human element of manually assembling these images is removed, providing a more robust and repeatable platform for image generation and subsequent comparison. Looking forward, using the automatic approach to produce full channel images of the same channel from different inspections, should allow a direct comparison of any changes in channel condition over time to be performed.

A software tool based on the method that has been described in this paper is currently being implemented for deployment at all AGR plants in the UK. The tool is being trialed and used initially by the expert operators who are currently assembling the defect montages by hand. It is anticipated that the tool will be used in parallel with the existing manual process until such times that the automatic approach is fully trusted by plant operators. In addition to automatically producing full channel images from inspection videos, the tool will highlight potential defect brick layers to a user and it will aim to automatically detect, locate and quantify any cracks or other anomalies in the channel. The system will be accompanied by a database which will allow users to instantly archive and access images which have been stitched by the tool. Furthermore, by accessing historic images in the database, users will be able to asses and quantify changes and developments of the channel conditions over time.

\section{ACKNOWLEDGEMENTS}

The authors would like to thank EDF Energy for funding this work. Thanks also to Rahul Summan and Gordon Dobie for various useful discussions about image stitching and camera calibration. 
[1] N. Houlton, "Life Extension of the EDF Energy Nuclear Fleet," Nuclear Institute Congress, Manchester, UK, Oct 2013.

[2] C. E. Watson, P. C. Robinson, and P. R. Maul, "The role of data visualisation in core inspection decision making," in Modelling and Measuring Reactor Core Graphite Properties and Performance, G. B. Neighbour, Ed., ed: The Royal Society of Chemistry, 2013, pp. 149-157.

[3] R. Szeliski, "Image mosaicing for tele-reality applications," in Proceedings of the Second IEEE Workshop on Applications of Computer Vision, 1994, pp. 44-53.

[4] H. Bay, A. Ess, T. Tuytelaars, and L. V. Gool, "Speeded-Up Robust Features (SURF)," Computer Vision and Image Understanding, vol. 110, pp. 346-359, Jun. 2008.

[5] H. Bay, T. Tuytelaars, and L. Van Gool, "SURF: Speeded Up Robust Features," in ECCV, 2006, pp. 404-417.

[6] M. Brown and D. G. Lowe, "Recognising panoramas," in Proc. ICCV 2003, Nice, France, 2003, pp. 1218-1225.

[7] T. Tuytelaars and K. Mikolajczyk, "Local invariant feature detectors: a survey," Foundations and Trends in Computer Graphics and Vision, vol. 3, pp. 177-280, 2008.

[8] R. Szeliski, "Image alignment and stitching a tutorial," Microsoft Research, Tech. Rep. MSR-TR-2004-92, Oct. 2004.

[9] P. Murray, G. West, S. Marshall, and S. McArthur, "Automated image stitching for enhanced visual inspections of nuclear power stations," in Proc. CM \& MFPT, Krakow, Poland, 2013.

[10] T. Bloodworth, M. Anderson, M. Brown, and J. Williams, "Eddy current inspection of AGR fuel channels: keyway cracks and density mapping," in Proc. NDT, Northamptonshire, UK, 2012.

[11] C. J. Wallace, G. M. West, S. D. J. McArthur, and D. Towle, "Distributed data and information fusion for nuclear reactor condition monitoring," IEEE Trans. Nuclear Science, vol. 59, pp. 182-189, Feb. 2012.

[12] A. Cole-Baker and J. Reed, "Measurement of AGR graphite fuel brick shrinkage and channel distortion," in Management of Ageing Processes in Graphite Cores. vol. 309, G. B. Neighbour, Ed., ed: Royal Society of Chemistry, 2007, pp. 201-208.

[13] G. Dobie, R. Summan, C. MacLeod, and S. Gareth Pierce, "Visual odometry and image mosaicing for NDE," NDT \& E International, vol. 57, pp. 17-25, Jul. 2013.

[14] R. Summan, "Positioning for mobile NDE inspection platforms," PhD Thesis, Dept. Electronic and Electrical Engineering, University of Strathclyde, Glasgow, Scotland, 2013.

[15] M. Brown and D. G. Lowe, "Automatic panoramic image stitching using invariant features," International Journal of Computer Vision, vol. 74, pp. 59-73, Aug. 2007.

[16] S. Mori, H. Nishida, and H. Yamada, Optical Character Recognition: John Wiley I\&amp; Sons, Inc., 1999.

[17] R. C. Gonzalez and R. E. Woods, Digital image processing. Upper Saddle River, NJ: Pearson Education, 2008.

[18] N. Otsu, "A threshold selection method from gray-level histograms," IEEE Transactions on Systems, Man and Cybernetics, vol. 9, pp. 62-66, Jan 1979.

[19] Z. Zhengyou, "A flexible new technique for camera calibration," Pattern Analysis and Machine Intelligence, IEEE Transactions on, vol. 22, pp. 1330-1334, 2000.

[20] D. G. Lowe, "Distinctive image features from scale-invariant keypoints," International Journal of Computer Vision, vol. 60, pp. 91-110, Nov. 2004. 
[21] M. A. Fischler and R. C. Bolles, "Random sample consensus: a paradigm for model fitting with applications to image analysis and automated cartography," Communications of the ACM, vol. 24, pp. 381-395, Jun. 1981. 